\title{
MINIMAX FRACTIONAL PROGRAMMING INVOLVING GENERALISED INVEX FUNCTIONS
}

\author{
H. C. $\mathrm{LAI}^{1}$ and J. C. $\mathrm{LIU}^{2}$
}

(Received 5 January, 1999; revised 30 October, 2000)

\begin{abstract}
The convexity assumptions for a minimax fractional programming problem of variational type are relaxed to those of a generalised invexity situation. Sufficient optimality conditions are established under some specific assumptions. Employing the existence of a solution for the minimax variational fractional problem, three dual models, the Wolfe type dual, the Mond-Weir type dual and a one parameter dual type, are constructed. Several duality theorems concerning weak, strong and strict converse duality under the framework of invexity are proved.
\end{abstract}

\section{Introduction}

Fractional programming is an interesting subject which features in several types of optimisation problems. For example, it can be used in engineering and economics to minimise a ratio of functions between a given period of time and a utilised resource in order to measure the efficiency or productivity of a system. In these sorts of problems the objective function is usually given as a ratio of functions in fractional programming form (see Stancu-Minasion [20]).

The optimisation problem considered in this paper consists of minimising a maximum of several time-dependent ratios involving integral expressions. Many authors have studied this type of variational programming (see for example $[1,4,5,8,19,21$, 22]). Other minimax programming problems involving generalised convexity are discussed in [12-15]. In this paper, the usual convexity assumptions are relaxed to those of a generalised invexity situation. Since the model involves a state function $x(t)$ in the integrand of the integration, the results also relate to questions of optimal control.

\footnotetext{
'Department of Applied Mathematics, Chung Yuang Christian University, Chung Li 320, Taiwan; e-mail: hclai@cycu.edu.tw.

${ }^{2}$ Section of Mathematics, National Overseas Chinese Student University, Linkou 24499 PO Box

1-1337, Taiwan.

(C) Australian Mathematical Society 2003, Serial-fee code 1446-1811/03
} 
We consider a minimax problem with a fractional objective in the form:

$$
\begin{gathered}
v^{*}=\min _{x} \max _{1 \leq i \leq p} \frac{\int_{a}^{b} f^{i}(t, x(t), \dot{x}(t)) d t}{\int_{a}^{b} g^{i}(t, x(t), \dot{x}(t)) d t} \\
\text { subject to } x \in P S\left(T, R^{n}\right), \quad x(a)=\alpha, \quad x(b)=\beta, \\
\int_{a}^{b} h^{j}(t, x(t), \dot{x}(t)) d t \leq 0, \\
j \in \underline{m} \equiv\{1,2, \ldots, m\}, \quad t \in T=[a, b],
\end{gathered}
$$

where the functions $f^{i}, g^{i}, i \in \underline{p}$ and $h^{j}, j \in \underline{m}$ are continuous in $t, x$ and $\dot{x}$ and have continuous partial derivatives with respect to $x$ and $\dot{x}$, and where $P S\left(T, R^{n}\right)$ is the space of all piecewise smooth state functions $x$ defined on the compact time set $T$ in $R$. The norm of $x \in P S\left(T, R^{n}\right)$ is defined by $\|x\|=\|x\|_{\infty}+\|D x\|_{\infty}$, where $D$ is the differential operator on $P S\left(T, R^{n}\right)$ defined by

$$
y=D x \quad \text { if and only if } \quad x(t)=x(a)+\int_{a}^{t} y(s) d s .
$$

So $D=d / d t$ except at the point of discontinuity. Throughout we assume that

$$
\int_{a}^{b} g^{i}(t, x(t), \dot{x}(t)) d t>0, \quad \int_{a}^{b} f^{i}(t, x(t), \dot{x}(t)) d t \geq 0
$$

for each $i \in \underline{p}$ and any $x \in \mathscr{F}_{P}$, the set of all feasible solutions of $(P)$. For simplicity, we write $x(t)=x$ and $\dot{x}(t)=\dot{x}$.

In order to obtain necessary and sufficient optimality conditions for problem $(P)$, Bector et al. [1] considered an equivalent parametric problem for $v \in R$ in the following form:

$\left(E P_{v}\right) \quad$ minimise $q$

$$
\begin{aligned}
& \text { subject to } x \in P S\left(T, R^{n}\right), \quad x(a)=\alpha, \quad x(b)=\beta, \\
& \qquad \int_{a}^{b}\left[f^{i}(t, x, \dot{x})-v g^{i}(t, x, \dot{x})\right] d t \leq q, \\
& \int_{a}^{b} h^{j}(t, x, \dot{x}) d t \leq 0, \quad \text { for } i \in \underline{p} \text { and } j \in \underline{m},
\end{aligned}
$$

and they established the following result.

LEMMA 1.1 ([1]). The function $x^{*}$ is an optimal solution of $(P)$ with optimal value $v^{*}$ if and only if the triple $\left(x^{*}, v^{*}, q^{*}\right)$ is an optimal solution of $\left(E P_{v}\right)$ with optimal value $q^{*}=0$. That is

$$
v^{*}=\frac{\int_{a}^{b} f^{i}\left(t, x^{*}, \dot{x}^{*}\right) d t}{\int_{a}^{b} g^{i}\left(t, x^{*}, \dot{x}^{*}\right) d t}
$$


Here all functions in $(P)$ are assumed to exhibit some generalised convexity. In [1], they also constructed various duality models for $\left(E P_{v}\right)$. In [16], Liu employed the same approach as in [1] to establish optimality conditions and duality theorems for $(P)$ in the case of generalised $(F, \rho)$-convex functions.

In this paper, we will establish optimality conditions and duality theorems in the case of generalised invexity in $(P)$ as well as in $\left(E P_{v}\right)$. To this end, in Section 2 we introduce some notation and preliminary results. In Section 3 , we give some definitions on generalised invexity and derive some sufficient optimality conditions for problem $(P)$ in the case of generalised invexity. Employing these results, we construct three dual problems in Sections 4-6. Here we investigate weak, strong and strict converse duality theorems under the framework of generalised invex functions.

\section{Notation and preliminary results}

For $x \in P S\left(T, R^{n}\right)$, we let $F^{i}(x)=\int_{a}^{b} f^{i}(t, x, \dot{x}) d t, G^{i}(x)=\int_{a}^{b} g^{i}(t, x, \dot{x}) d t$ and $H^{j}(x)=\int_{a}^{b} h^{j}(t, x, \dot{x}) d t$, for $i \in \underline{p}$ and $j \in \underline{m}$. Suppose that functions $f^{i}, g^{i}$ and $h^{j}$ are continuous in $t, x$ and $\dot{x}$ and have continuous partial derivatives with respect to (w.r.t.) $x$ and $\dot{x}$. Then the functionals $F=\left(F^{1}, F^{2}, \ldots, F^{p}\right), G=\left(G^{1}, G^{2}, \ldots, G^{p}\right)$ and $H=\left(H^{1}, H^{2}, \ldots, H^{m}\right)$ are (Fréchet) differentiable on $P S\left(T, R^{n}\right)$. It follows that the problem $(P)$ may be rewritten in the form:

$$
\begin{aligned}
& (P) \quad \min _{x \in P S\left(T, R^{n}\right)} \max _{i \in \underline{\underline{p}}}\left(\frac{F^{i}(x)}{G^{i}(x)}\right) \\
& \text { subject to } x(a)=\alpha, x(b)=\beta \text { and } H(x) \leq 0 .
\end{aligned}
$$

Here $\alpha$ and $\beta$ are fixed vectors in $R^{n}$. The equivalent parametric minimisation problem $\left(E P_{v}\right)$ is then given by

$$
\begin{aligned}
\left(E P_{v}\right) \quad \text { minimise } & q \\
\text { subject to } & F^{i}(x)-v G^{i}(x) \leq q, i \in \underline{p}, \quad H^{j}(x) \leq 0, j \in \underline{m}, \\
& x \in P S\left(T, R^{n}\right) \text { with fixed boundary conditions } \\
& x(a)=\alpha \text { and } x(b)=\beta .
\end{aligned}
$$

As in $[4,5]$, it can be shown that if $x \in \mathscr{F}_{P}$, a feasible solution of $(P)$, then

$$
\phi(x) \equiv \max _{i \in \underline{p}} \frac{F^{i}(x)}{G^{i}(x)}=\max _{\substack{\langle y, e\rangle=1 \\ y \in R_{+}^{p}}} \frac{\langle y, F(x)\rangle}{\langle y, G(x)\rangle},
$$

where $e$ is a vector of ones in $R_{+}^{p}$ and $\langle\cdot, \cdot\rangle$ denotes the inner product in $R^{p}$. For convenience, henceafter we define $I=\left\{y \in R_{+}^{p} \mid\langle y, e\rangle=1\right\}$. 
For simplicity, for $x \in P S\left(T, R^{n}\right), y \in R_{+}^{p}$ and $z \in R_{+}^{m}$ we denote

$$
\begin{aligned}
& \Phi(x, y)=\langle y, F(x)\rangle=\int_{a}^{b} \sum_{i=1}^{p} y^{i} f^{i}(t, x, \dot{x}) d t, \\
& \Psi(x, y)=\langle y, G(x)\rangle=\int_{a}^{b} \sum_{i=1}^{p} y^{i} g^{i}(t, x, \dot{x}) d t \text { and } \\
& \Omega(x, z)=\langle y, H(x)\rangle=\int_{a}^{b} \sum_{j=1}^{m} z^{j} h^{j}(t, x, \dot{x}) d t .
\end{aligned}
$$

Evidently $\Phi(x, \cdot), \Psi(x, \cdot)$ and $\Omega(x, \cdot)$ are linear functionals.

From (2.1), if $x^{*}$ is an optimal solution of $(P)$, then

$$
\begin{aligned}
\phi\left(x^{*}\right) & =\max _{i \in \underline{p}} \frac{F^{i}\left(x^{*}\right)}{G^{i}\left(x^{*}\right)}=\max _{y \in I} \frac{\Phi\left(x^{*}, y\right)}{\Psi\left(x^{*}, y\right)} \\
& =\frac{\Phi\left(x^{*}, \hat{y}\left(x^{*}\right)\right)}{\Psi\left(x^{*}, \hat{y}\left(x^{*}\right)\right)}=\min _{x} \max _{y \in I} \frac{\Phi(x, y)}{\Psi(x, y)}=\frac{\Phi\left(x^{*}, y^{*}\right)}{\Psi\left(x^{*}, y^{*}\right)},
\end{aligned}
$$

where $\hat{y}\left(x^{*}\right)=y^{*}$.

Problem $(P)$ is actually equivalent to

$$
\min _{x} \max _{y} \frac{\Phi(x, y)}{\Psi(x, y)} \quad \text { subject to } H(x) \leq 0, x \in R^{n} \quad \text { and } \quad y \in I .
$$

The concept used here for the solution of $\left(E P_{v}\right)$ coincides with finding the minimax solution of the Lagrangian

$$
\begin{aligned}
L(x, y ; v, z) & =\langle y, F(x)\rangle-v\langle y, G(x)\rangle+\langle z, H(x)\rangle \\
& =\Phi(x, y)-v \Psi(x, y)+\Omega(x, z)
\end{aligned}
$$

for multipliers $v^{*} \in R, z \in R_{+}^{m}$, and the solution is given by $L^{\prime}(x, y) \xi=0$ for any $\xi \in C\left([a, b], R^{n}\right)$. Hence necessary optimality conditions for $(P)$, as in Bector et al. [2, Theorem 1] (see also Craven [7]) are actually Kuhn-Tucker type conditions which we can rewrite as follows.

THEOREM 2.1 (Necessary conditions). If $x^{*}$ is an optimal solution of $(P)$, then there exist $y^{*} \in I$ and multipliers $v^{*} \in R$ and $z^{*} \in R_{+}^{m}$ such that $\left(x^{*}, v^{*}, y^{*}, z^{*}\right)$ satisfies

$$
\begin{aligned}
\Phi^{\prime}\left(x^{*}, y^{*}\right)-v^{*} \Psi^{\prime}\left(x^{*}, y^{*}\right)+\Omega^{\prime}\left(x^{*}, z^{*}\right) & =0, \\
\Phi\left(x^{*}, y^{*}\right)-v^{*} \Psi\left(x^{*}, y^{*}\right) & =0, \\
\Omega\left(x^{*}, z^{*}\right) & =0,
\end{aligned}
$$

where $\Phi^{\prime}$ and $\Psi^{\prime}$ are the gradients of $\Phi$ and $\Psi$ at $\left(x^{*}, y^{*}\right)$ respectively and $\Omega^{\prime}\left(x^{*}, z^{*}\right)=$ $\left\langle z^{*}, \nabla H(x)\right\rangle$. 
In order to construct parameter-free dual models for $(P)$, using $(2.4)$ we replace $v^{*}$ by $\Phi\left(x^{*}, y^{*}\right) / \Psi\left(x^{*}, y^{*}\right)$, and then restate Theorem 2.1 as follows.

THEOREM 2.2 (Necessary condition). If $x^{*}$ is an optimal solution of $(P)$, then there exist $y^{*} \in I$ and multipliers $v^{*} \in R$ and $z^{*} \in R_{+}^{m}$ such that $\left(x^{*}, y^{*}, z^{*}\right)$ satisfies

$$
\begin{aligned}
\Psi\left(x^{*}, y^{*}\right) \Phi_{1}^{\prime}\left(x^{*}, y^{*}\right)-\Phi\left(x^{*}, y^{*}\right) \Psi_{1}^{\prime}\left(x^{*}, y^{*}\right)+\Psi\left(x^{*}, y^{*}\right) \Omega_{1}^{\prime}\left(x^{*}, z^{*}\right)=0, \\
\Omega\left(x^{*}, z^{*}\right)=0,
\end{aligned}
$$

and obtain the optimal value by

$$
\phi\left(x^{*}\right)=\frac{\Phi\left(x^{*}, y^{*}\right)}{\Psi\left(x^{*}, y^{*}\right)}=\max _{y \in I} \frac{\Phi\left(x^{*}, y\right)}{\Psi\left(x^{*}, y\right)}\left(=\min _{x} \max _{1 \leq i \leq p} \frac{F^{i}(x)}{\left.G^{i}(x)\right)}\right) .
$$

\section{Generalised invexity}

For sufficient optimality conditions of the fractional variational problem $(P)$, we will relax the assumption of convexity to that of generalised invexity (see Mond and Husain [19]). This approach will give a basic technique for constructing some duality theorems for problem $(P)$.

Let $x \in P S\left(T, R^{n}\right)$ and $f \in C^{1}\left(T \times R^{n} \times R^{n}\right)$. Define a functional $J: P S\left(T, R^{n}\right) \mapsto R$ by $J(x)=\int_{a}^{b} f(t, x, \dot{x}) d t$.

We assume that the boundary points $x(a)$ and $x(b)$ are fixed. Consider the admissible vectors $x+w$ with admissible variations $w \in C\left([a, b], R^{n}\right)$ vanishing at the boundary points. Then the differential of $J$ is a linear functional on $C\left([a, b], R^{n}\right)$,

$$
\begin{aligned}
J^{\prime}(x) w & =\left.\frac{d}{d \alpha} \int_{a}^{b} f(t, x+\alpha w, \dot{x}+\alpha \dot{w}) d t\right|_{\alpha=0} \\
& =\int_{a}^{b}\left[f_{x}(t, x, \dot{x}) w(t)+f_{\dot{x}}(t, x, \dot{x}) \dot{w}(t)\right] d t \\
& =\int_{a}^{b}\left[f_{x}(t, x, \dot{x})-D f_{\dot{x}}(t, x, \dot{x})\right] w(t) d t+\left.f_{\dot{x}}(t, x, \dot{x}) w(t)\right|_{a} ^{b} \\
& =\int_{a}^{b}\left[f_{x}(t, x, \dot{x})-D f_{\dot{x}}(t, x, \dot{x})\right] w(t) d t .
\end{aligned}
$$

That is,

$$
J^{\prime}(x) w=\int_{a}^{b}\left[f_{x}(t, x, \dot{x})-D f_{\dot{x}}(t, x, \dot{x})\right] w(t) d t
$$

for all $w \in C\left([a, b], R^{n}\right), w(a)=0=w(b)$, where $D=d / d t$. 
This concept allows us to define a function $\eta: P S\left(T, R^{n}\right) \times P S\left(T, R^{n}\right) \mapsto$ $C\left(T, R^{n}\right)$ with condition $\eta(x, u)=0$ if $x=u$. Using this function $\eta$, we will give the following definitions for generalised invexity.

DEFINITION 3.1. For any $u \in P S\left(T, R^{n}\right)$,

(i) a differentiable function $J$ is said to be invex w.r.t. $\eta$ if

$$
J(x)-J(u) \geq J^{\prime}(u) \eta(x, u)
$$

(ii) $J$ is said to be pseudoinvex w.r.t. $\eta$ if $J^{\prime}(u) \eta(x, u) \geq 0 \Rightarrow J(x) \geq J(u)$, or equivalently $J(x)<J(u) \Rightarrow J^{\prime}(u) \eta(x, u)<0$;

(iii) $J$ is said to be strictly pseudoinvex w.r.t. $\eta$ if, for $x \neq u$,

$$
J^{\prime}(u) \eta(x, u) \geq 0 \Rightarrow J(x)>J(u),
$$

or equivalently $J(x) \leq J(u) \Rightarrow J^{\prime}(u) \eta(x, u)<0$;

(iv) $J$ is said to be quasi-invex w.r.t. $\eta$ if

$$
J^{\prime}(u) \eta(x, u)>0 \Rightarrow J(x)>J(u),
$$

or equivalently $J(x) \leq J(u) \Rightarrow J^{\prime}(u) \eta(x, u) \leq 0$.

REMARK 3.1. If $x, u \in \mathscr{F}_{P}$, then $x(a)=u(a)=\alpha, x(b)=u(b)=\beta$ and so $\eta(x(a), u(a))=0, \eta(x(b), u(b))=0$. This shows that the function $\eta(x, u)$ on $T$ satisfies the zero boundary condition. It follows that in (3.2) of Definition 3.1 if $x, u \in \mathscr{F}_{P}$ we always have the representation (3.1):

$$
J^{\prime}(u) \eta(x, u)=\int_{a}^{b}\left[f_{x}(t, u, \dot{u})-D f_{\dot{x}}(t, u, \dot{u})\right] \eta(x, u)(t) d t,
$$

where $\eta(x, u) \in C\left(T, R^{n}\right)$.

This shows that the concept of generalised invexity used here actually coincides with the original concept of invexity given by Hanson and Mond [9]. For details, consult Craven [8].

For convenience, we denote by $\Phi_{1}^{\prime}(x, y)=\Phi_{x}(x, y), \Psi_{1}^{\prime}(x, y)=\Psi_{x}(x, y)$ the partial Fréchet derivative with respect to the feasible variable $x$.

Now we can state sufficient optimality conditions for $(P)$ in the case of generalised invexity as follows.

THEOREM 3.1 (Sufficient conditions). Let $x^{*} \in \mathscr{F}_{P}, y^{*} \in I, z^{*} \in R_{+}^{m}$ and $\left(x^{*}, y^{*}, z^{*}\right)$ satisfy (2.6)-(2.8). Define $A(x)=\Psi\left(x^{*}, y^{*}\right) \Phi\left(x, y^{*}\right)-\Psi\left(x, y^{*}\right) \Phi\left(x^{*}, y^{*}\right)$ and $B(x)=A(x)+\Psi\left(x^{*}, y^{*}\right) \Omega\left(x, z^{*}\right)$. If, for the given $y^{*}$ and $z^{*}$, any one of the following conditions holds: 
(a) $\Phi(\cdot, y *),-\Psi\left(\cdot, y^{*}\right)$ and $\Omega\left(\cdot, z^{*}\right)$ are invex w.r.t. the function $\eta$;

(b) $A$ is pseudoinvex and $\Omega\left(\cdot, z^{*}\right)$ is quasi-invex w.r.t. $\eta$;

(c) $A$ is quasi-invex and $\Omega\left(\cdot, z^{*}\right)$ is strictly pseudoinvex w.r.t. $\eta$;

(d) $B$ is pseudoinvex w.r.t. $\eta$,

then $x^{*}$ is an optimal solution of $(P)$.

PROOF. If $x^{*}$ is not an optimal solution of $(P)$, then there is a feasible solution $u \in \mathscr{F}_{P}$ such that

$$
\phi\left(x^{*}\right)>\phi(u)
$$

By (2.1) and (2.2),

$$
\frac{\Phi\left(x^{*}, y^{*}\right)}{\Psi\left(x^{*}, y^{*}\right)}=\phi\left(x^{*}\right)>\phi(u)=\max _{y \in I} \frac{\Phi(u, y)}{\Psi(u, y)} \geq \frac{\Phi\left(u, y^{*}\right)}{\Psi\left(u, y^{*}\right)}, \quad y^{*} \in I .
$$

It follows that

$$
A(u)=\Phi\left(u, y^{*}\right) \Psi\left(x^{*}, y^{*}\right)-\Phi\left(x^{*}, y^{*}\right) \Psi\left(u, y^{*}\right)<0=A\left(x^{*}\right)
$$

and so by rearranging the above inequality, we get

$$
\Psi\left(x^{*}, y^{*}\right)\left[\Phi\left(u, y^{*}\right)-\Phi\left(x^{*}, y^{*}\right)\right]-\Phi\left(x^{*}, y^{*}\right)\left[\Psi\left(u, y^{*}\right)-\Psi\left(x^{*}, y^{*}\right)\right]<0 .
$$

Since $u \in \mathscr{F}_{P}$, by condition (2.6), we have

$$
\Omega\left(u, z^{*}\right) \leq 0=\Omega\left(x^{*}, z^{*}\right) .
$$

Consequently, (3.5) and (3.7) yield $B(u)<B\left(x^{*}\right)$. Now if condition (a) holds, for $y^{*} \in I, z^{*} \in R_{+}^{m}$, employing the invexity of $\Phi\left(\cdot, y^{*}\right),-\Psi\left(\cdot, y^{*}\right)$ and $\Omega\left(\cdot, z^{*}\right)$; we then have the inequalities

$$
\begin{aligned}
\Phi\left(u, y^{*}\right)-\Phi\left(x^{*}, y^{*}\right) & \geq \Phi_{1}^{\prime}\left(x^{*}, y^{*}\right) \eta\left(u, x^{*}\right), \\
-\left[\Psi\left(u, y^{*}\right)-\Psi\left(x^{*}, y^{*}\right)\right] & \geq-\Psi_{1}^{\prime}\left(x^{*}, y^{*}\right) \eta\left(u, x^{*}\right), \\
\Omega\left(u, z^{*}\right)-\Omega\left(x^{*}, z^{*}\right) & \geq \Omega_{1}^{\prime}\left(x^{*}, z^{*}\right) \eta\left(u, x^{*}\right) .
\end{aligned}
$$

Here for each $y^{*}$ and $z^{*}, \Phi_{1}^{\prime}, \Psi_{1}^{\prime}$ and $\Omega_{1}^{\prime}$ are partial Fréchet derivatives of $\Phi\left(\cdot, y^{*}\right)$, $-\Psi\left(\cdot, y^{*}\right)$ and $\Omega\left(\cdot, z^{*}\right)$ at $x^{*}$, respectively. Since $\Psi\left(x^{*}, y^{*}\right)>0$ and $\Phi\left(x^{*}, y^{*}\right) \geq 0$, we multiply (3.8) by $\Psi\left(x^{*}, y^{*}\right),(3.9)$ by $\Phi\left(x^{*}, y^{*}\right)$ and (3.10) by $\Psi\left(x^{*}, y^{*}\right)$. Adding up the resulting inequalities, it follows from (3.6) and (3.7) that

$$
0>\left[\Psi\left(x^{*}, y^{*}\right) \Phi_{1}^{\prime}\left(x^{*}, y^{*}\right)-\Phi\left(x^{*}, y^{*}\right) \Psi_{1}^{\prime}\left(x^{*}, y^{*}\right)+\Psi\left(x^{*}, y^{*}\right) \Omega_{1}^{\prime}\left(x^{*}, z^{*}\right)\right] \eta\left(u, x^{*}\right) .
$$


This contradicts (2.6). Hence (3.4) does not hold, and so $x^{*}$ must be optimal for $(P)$.

For condition (b), $A$ is pseudoinvex and so from (3.5),

$$
A(u)<A\left(x^{*}\right) \Rightarrow A^{\prime}\left(x^{*}\right) \eta\left(u, x^{*}\right)<0,
$$

that is,

$$
\left[\Psi\left(x^{*}, y^{*}\right) \Phi_{1}^{\prime}\left(x^{*}, y^{*}\right)-\Phi\left(x^{*}, y^{*}\right) \Psi_{1}^{\prime}\left(x^{*}, y^{*}\right)\right] \eta\left(u, x^{*}\right)<0 .
$$

The identity (2.6) and inequality (3.11) yield $\Omega_{1}^{\prime}\left(x^{*}, z^{*}\right) \Psi\left(x^{*}, y^{*}\right) \eta\left(u, x^{*}\right)>0$. Since $\Psi\left(x^{*}, y^{*}\right)>0$, it follows that

$$
\Omega_{1}^{\prime}\left(x^{*}, z^{*}\right) \eta\left(u, x^{*}\right)>0 .
$$

On the other hand, the function $\Omega$ in condition (b) is assumed to be quasi-invex w.r.t. $\eta$. Thus inequality (3.12) implies $\Omega_{1}^{\prime}\left(x^{*}, z^{*}\right) \eta\left(u, x^{*}\right) \leq 0$, (by (3.3)), which contradicts (3.12). Hence (3.4) does not hold. This shows that $x^{*}$ is optimal for $(P)$.

If conditions (c) or (d) hold, the optimality of $x^{*}$ can be proved using the same argument as that for condition (b). Hence the proof is complete.

In the next sections, we will construct some dual models.

\section{The first dual model-the Wolfe type dual}

Employing Theorem 2.2, we will construct two parametric-free dual models. Consider the Wolfe type dual problem given by

$$
\begin{aligned}
& \text { (D1) Maximise } \frac{\Phi(u, y)+\Omega(u, z)}{\Psi(u, y)} \\
& \text { subject to }(u, z) \in P S\left(T, R^{n}\right) \times R_{+}^{m} \text { and } y \in I \subset R_{+}^{m}, \\
& u(a)=\alpha, \quad u(b)=\beta, \\
& \Psi(u, y) \Phi_{1}^{\prime}(u, y)-[\Phi(u, y)+\Omega(u, z)] \Psi_{1}^{\prime}(u, y) \\
& +\Psi(u, y) \Omega_{1}^{\prime}(u, z)=0 .
\end{aligned}
$$

Denote by $K_{1}$ the set of all feasible solutions of problem $(D 1)$. We assume throughout this section that

$$
\Phi(u, y)+\Omega(u, z) \geq 0 \text { and } \Psi(u, y)>0 \text { for all }(u, y, z) \in K_{1} .
$$

In what follows, we shall establish weak, strong and strict converse duality theorems for $(P)-(D 1)$. 
THEOREM 4.1 (Weak duality). Let $x \in \mathscr{F}_{p},(u, y, z) \in K_{1}$ and let

$$
C(x)=\Psi(u, y)[\Phi(x, y)+\Omega(x, z)]-[\Phi(u, y)+\Omega(u, z)] \Psi(x, y) .
$$

If, for each $y$ and $z$, either one of the following conditions holds:

(a) $\Phi\left(\cdot, y^{*}\right),-\Psi\left(\cdot, y^{*}\right)$ and $\Omega\left(\cdot, z^{*}\right)$ are invex w.r.t. the function $\eta$ (defined as in Definition 3.1);

(b) C is pseudoinvex w.r.t. $\eta$.

then

$$
\phi(x) \geq \frac{\Phi(u, y)+\Omega(u, z)}{\Psi(u, y)},
$$

where $\phi(x)$ is defined by (2.1).

PROOF. If (4.3) were not true, then

$$
\phi(x)<\frac{\Phi(u, y)+\Omega(u, z)}{\Psi(u, y)} .
$$

It follows from (2.2) that for any $y \in I$,

or

$$
\frac{\Phi(x, y)}{\Psi(x, y)} \leq \max _{\beta \in I} \frac{\Phi(x, \beta)}{\Psi(x, \beta)}=\phi(x)<\frac{\Phi(u, y)+\Omega(u, z)}{\Psi(u, y)},
$$

$$
\Phi(x, y) \Psi(u, y)-[\Phi(u, y)+\Omega(u, z)] \Psi(x, y)<0 .
$$

Rewriting the above inequality, we get

$$
[\Phi(x, y)+\Omega(x, z)] \Psi(u, y)-[\Phi(u, y)+\Omega(u, z)] \Psi(x, y)<\Omega(x, z) \Psi(u, y) .
$$

Since $\Omega(x, z) \leq 0$, if $x \in \mathscr{F}_{P}, z \in R_{+}^{m}$ and $\Psi(u, y)>0, \Omega(x, z) \Psi(u, y) \leq 0$. It follows from (4.6) that

$$
C(x)<0=C(u)
$$

Now if condition (a) holds, for each $y \in I, z \in R_{+}^{m}$, the invexity of $\Phi\left(\cdot, y^{*}\right)$, $-\Psi\left(\cdot, y^{*}\right)$ and $\Omega\left(\cdot, z^{*}\right)$ implies that

$$
\begin{aligned}
\Phi(x, y)-\Phi(u, y) & \geq \Phi_{1}^{\prime}(u, y) \eta(x, u), \\
-[\Psi(x, y)-\Psi(u, y)] & \geq-\Psi_{1}^{\prime}(u, y) \eta(x, u), \\
\Omega(x, z)-\Omega(u, z) & \geq \Omega_{1}^{\prime}(u, z) \eta(x, u),
\end{aligned}
$$


or

$$
-\Omega(u, z) \geq-\Omega(x, z)+\Omega_{1}^{\prime}(u, z) \eta(x, u) .
$$

From (4.2), $\Psi(u, y)$ and $\Phi(u, y)+\Omega(u, z)$ are nonnegative. Multiplying (4.8) by $\Psi(u, y),(4.9)$ by $\Phi(u, y)+\Omega(u, z)$ and (4.10) by $\Psi(u, y)$, and summing up the resulting inequalities, then from $(4.5)$ we eventually obtain that

$$
\left\{\Psi(u, y) \Phi_{1}^{\prime}(u, y)-[\Phi(u, y)+\Omega(u, z)] \Psi_{1}^{\prime}(u, y)+\Psi(u, y) \Omega_{1}^{\prime}(u, z)\right\} \eta(x, u)<0
$$

This contradicts (4.1). So (4.4) does not hold and (4.2) does hold.

From condition (b), $C$ is pseudoinvex and (4.7) implies that $C^{\prime}(u) \eta(x, u)<0$, that is, $\left\{\Psi(u, y) \Phi_{1}^{\prime}(u, y)-[\Phi(u, y)+\Omega(u, z)] \Psi_{1}^{\prime}(u, y)+\Psi(u, y) \Omega_{1}^{\prime}(u, z)\right\} \eta(x, u)<0$. This contradicts (4.1). Hence (4.3) does not hold and the proof is complete.

THEOREM 4.2 (Strong duality). If $x^{*}$ is an optimal solution of $(P)$ satisfying the conditions of Theorems 2.2 and 4.1, then there exist $y^{*} \in I$ and $z^{*} \in R_{+}^{m}$ such that $\left(x^{*}, y^{*}, z^{*}\right)$ is an optimal solution of $(D 1)$ and the optimal values of $(P)$ and $(D 1)$ are equal; that is, $\min (P)=\max (D 1)$.

PROOF. If $x^{*}$ is an optimal solution of $(P)$, then by Theorem 2.2, there exist $y^{*} \in I$ and $z^{*} \in R_{+}^{m}$ which satisfy the constraints of $(D 1)$, so that $\left(x^{*}, y^{*}, z^{*}\right) \in K_{1}$. Furthermore

$$
\frac{\Phi\left(x^{*}, y^{*}\right)+\Omega\left(x^{*}, z^{*}\right)}{\Psi\left(x^{*}, y^{*}\right)}=\frac{\Phi\left(x^{*}, y^{*}\right)}{\Psi\left(x^{*}, y^{*}\right)}=\phi\left(x^{*}\right),
$$

since $\Omega\left(x^{*}, z^{*}\right)=0$. Hence $\left(x^{*}, y^{*}, z^{*}\right)$ is an optimal solution of $(D 1)$. Consequently, (4.3) demonstrates that $(P)$ and $(D 1)$ have the same optimal values.

THEOREM 4.3 (Strict converse duality). Let $x_{1}$ and $\left(x^{*}, y_{0}, z_{0}\right)$ be optimal solutions for $(P)$ and $(D 1)$, respectively. Assume that the assumptions of Theorem 4.2 are fulfilled, and that

$$
C(\cdot)=\Psi\left(x^{*}, y_{0}\right)\left[\Phi\left(\cdot, y_{0}\right)+\Omega\left(\cdot, z_{0}\right)\right]-\left[\Phi\left(x^{*}, y_{0}\right)+\Omega\left(x^{*}, z_{0}\right)\right] \Psi\left(\cdot, y_{0}\right)
$$

is a strictly pseudoinvex function w.r.t. $\eta$. Then $x_{1}=x^{*}$ is an optimal solution of $(P)$, and $(P)$ and $(D 1)$ have the same optimal value

$$
\phi\left(x_{1}\right)=\frac{\Phi\left(x^{*}, y_{0}\right)+\Omega\left(x^{*}, z_{0}\right)}{\Psi\left(x^{*}, y_{0}\right)} .
$$


PROOF. Suppose on the contrary that $x_{1} \neq x^{*}$. Then by Theorem 4.2, there exist $y_{1} \in I$ and $z_{1} \in R_{+}^{m}$ such that $\left(x_{1}, y_{1}, z_{1}\right)$ is an optimal solution of $(D 1)$ and

$$
\phi\left(x_{1}\right)=\frac{\Phi\left(x_{1}, y_{1}\right)+\Omega\left(x_{1}, z_{1}\right)}{\Psi\left(x_{1}, y_{1}\right)} .
$$

As in the proof given in Theorem 4.1, we replace $x$ by $x_{1}$, and $(u, y, z)$ by $\left(x^{*}, y_{0}, z_{0}\right)$, to derive the inequality

$$
\phi\left(x_{1}\right)>\frac{\Phi\left(x^{*}, y_{0}\right)+\Omega\left(x^{*}, z_{0}\right)}{\Psi\left(x^{*}, y_{0}\right)} .
$$

This contradicts the fact that

$$
\phi\left(x_{1}\right)=\frac{\Phi\left(x_{1}, y_{1}\right)+\Omega\left(x_{1}, z_{1}\right)}{\Psi\left(x_{1}, y_{1}\right)}=\frac{\Phi\left(x^{*}, y_{0}\right)+\Omega\left(x^{*}, z_{0}\right)}{\Psi\left(x^{*}, y_{0}\right)} .
$$

Hence we conclude that

$$
x_{1}=x^{*} \text { and } \phi\left(x_{1}\right)=\frac{\Phi\left(x^{*}, y_{0}\right)+\Omega\left(x^{*}, z_{0}\right)}{\Psi\left(x^{*}, y_{0}\right)} .
$$

\section{The second dual model-the Mond-Weir type dual}

We introduce the Mond-Weir type dual problem as follows:

$$
\begin{aligned}
& \text { (D2) } \quad \text { Maximise } \frac{\Phi(u, y)}{\Psi(u, y)} \\
& \text { subject to }(u, y) \in P S\left(T, R^{n}\right) \times I, \quad u(a)=\alpha, \quad u(b)=\beta, \\
& \Psi(u, y) \Phi_{1}^{\prime}(u, y)-\Phi(u, y) \Psi_{1}^{\prime}(u, y)+\Psi(u, y) \Omega_{1}^{\prime}(u, z)=0, \\
& \\
& \Omega(u, z) \geq 0, z \in R_{+}^{m} .
\end{aligned}
$$

Denote by $K_{2}$ the set of all feasible solutions of problem (D2). We still assume throughout that $\Phi(u, y) \geq 0$ and $\Psi(u, y)>0$ for all $(u, y, z) \in K_{2}$. We will establish weak, strong and strict converse duality theorems for $(P)-(D 2)$.

THEOREM 5.1 (Weak duality). Let $x \in \mathscr{F}_{P},(u, y, z) \in K_{2}$ and define

$$
\begin{aligned}
& D(x)=\Psi(u, y) \Phi(x, y)-\Psi(x, y) \Phi(u, y) \text { and } \\
& E(x)=D(x)+\Psi(u, y) \Omega(x, z) .
\end{aligned}
$$

If, for each $y \in I, z \in R_{+}^{m}$, any one of the following conditions holds:

(a) $\Phi(\cdot, y),-\Psi(\cdot, y)$ and $\Omega(\cdot, z)$ are invex w.r.t. $\eta$; 
(b) $D$ is pseudoinvex and $\Omega(\cdot, z)$ is quasi-invex w.r.t. $\eta$;

(c) $D$ is quasi-invex and $\Omega(\cdot, z)$ is strictly pseudoinvex w.r.t. $\eta$;

(d) $E$ is pseudoinvex w.r.t. $\eta$,

then

$$
\phi(x) \geq \frac{\Phi(u, y)}{\Psi(u, y)} .
$$

ProOF. Suppose the result of (5.3) were not true. Then

$$
\frac{\Phi(u, y)}{\Psi(u, y)}>\phi(x)=\max _{y_{1} \in I} \frac{\Phi\left(x, y_{1}\right)}{\Psi\left(x, y_{1}\right)} \geq \frac{\Phi(x, y)}{\Psi(x, y)} .
$$

This implies that

$$
D(x)=\Phi(x, y) \Psi(u, y)-\Phi(u, y) \Psi(x, y)<0=D(u) .
$$

As $x \in \mathscr{F}_{P}, z \in R_{+}^{m}$ and $\Omega(x, z) \leq 0$, the constraint inequality (5.2) of (D2) yields

$$
\Omega(x, z) \leq 0 \leq \Omega(u, z) .
$$

Consequently, (5.4) and (5.5) yield

$$
E(x)<E(u) .
$$

If condition (a) holds, then by the invexity of $\Phi,-\Psi$ and $\Omega$, we have

$$
\begin{aligned}
\Phi(x, y)-\Phi(u, y) & \geq \Phi_{1}^{\prime}(u, y) \eta(x, u), \\
-[\Psi(x, y)-\Psi(u, y)] & \geq-\Psi_{1}^{\prime}(u, y) \eta(x, u), \\
\Omega(x, z)-\Omega(u, z) & \geq \Omega^{\prime}(u, z)_{1} \eta(x, u) .
\end{aligned}
$$

Since $\Psi(u, y)$ and $\Phi(u, y)$ are nonnegative, we multiply (5.7) by $\Psi(u, y),(5.8)$ by $\Phi(u, y)$ and (5.9) by $\Psi(u, y)$, and sum up the resulting inequalities. Eventually, from (5.4) and (5.5), we obtain the inequality

$$
0>\left[\Psi(u, y) \Phi_{1}^{\prime}(u, y)-\Phi(u, y) \Psi_{1}^{\prime}(u, y)+\Psi(u, y) \Omega_{1}^{\prime}(u, z)\right] \eta(x, u) .
$$

This contradicts (5.1) in the constraint of (D2). Hence (5.3) holds.

In condition (b), $D$ is pseudoinvex. Thus (5.4) implies $D^{\prime}(u) \eta(x, u)<0$, that is,

$$
\left[\Psi(u, y) \Phi_{1}^{\prime}(u, y)-\Phi(u, y) \Psi_{1}^{\prime}(u, y)\right] \eta(x, u)<0 .
$$

It follows from (5.1) and (5.10) that

$$
-\Psi(u, y) \Omega_{1}^{\prime}(u, z) \eta(x, u)<0 \quad \text { or } \quad \Omega_{1}^{\prime}(u, z) \eta(x, u)>0
$$


since $\Psi(u, y)>0$. But $\Omega$ is quasi-invex, so the above inequality implies

$$
\Omega(x, z)>\Omega(u, z)
$$

which contradicts (5.5). So (5.3) holds.

The proof for the cases when conditions (c) or (d) hold is similar. Hence the proof is complete.

THEOREM 5.2 (Strong duality). If $x^{*}$ is an optimal solution of $(P)$ satisfying the conditions of Theorems 2.2 and 5.1, then there exist $y^{*} \in I$ and $z^{*} \in R_{+}^{m}$ such that $\left(x^{*}, y^{*}, z^{*}\right)$ is an optimal solution of $(D 2)$, and $(P)$ and $(D 2)$ have the same optimal values.

ProOF. If $x^{*}$ is an optimal solution of $(P)$, then by Theorem 2.2, there exist $y^{*} \in I$ and $z^{*} \in R_{+}^{m}$ such that $\left(x^{*}, y^{*}, z^{*}\right) \in K_{2}$, the feasible solution of $(D 2)$, and

$$
\phi\left(x^{*}\right)=\frac{\Phi\left(x^{*}, y^{*}\right)}{\Psi\left(x^{*}, y^{*}\right)}=\max _{y \in I} \frac{\Phi\left(x^{*}, y\right)}{\Psi\left(x^{*}, y\right)} .
$$

It follows from Theorem 5.1 that $\left(x^{*}, y^{*}, z^{*}\right)$ is an optimal solution of $(D 2)$ and $\min (P)=\max \left(D_{2}\right)$.

THEOREM 5.3 (Strict converse duality). Let $x_{1}$ and $\left(x^{*}, y_{0}, z_{0}\right)$ be optimal solutions of $(P)$ and (D2), respectively. Assume that the conditions of Theorem 5.2 are fulfilled, and that $D(\cdot)=\Psi\left(x^{*}, y_{0}\right) \Phi\left(\cdot, y_{0}\right)-\Psi\left(\cdot, y_{0}\right) \Phi\left(x^{*}, y_{0}\right)$ is strictly pseudoinvex and $\Omega\left(\cdot, z_{0}\right)$ is quasi-invex w.r.t. $\eta$. Then $x_{1}=x^{*}$ is an optimal solution of $(P)$, and $(P)$ and $(D 2)$ have the same optimal values $\phi\left(x_{1}\right)=\Phi\left(x^{*}, y_{0}\right) / \Psi\left(x^{*}, y_{0}\right)$.

PROOF. Since $x_{1}$ is an optimal solution of $(P)$, by Theorem 5.2, there exist $y_{1} \in I$ and $z_{1} \in R_{+}^{m}$ such that $\left(x_{1}, y_{1}, z_{1}\right)$ is an optimal solution of $(D 2)$ and

$$
\phi\left(x_{1}\right)=\frac{\Phi\left(x_{1}, y_{1}\right)}{\Psi\left(x_{1}, y_{1}\right)} .
$$

On the other hand, $\left(x^{*}, y_{0}, z_{0}\right)$ is an optimal solution of $(D 2)$, with optimal value equal to $\phi\left(x_{1}\right)$, that is,

$$
\phi\left(x_{1}\right)=\frac{\Phi\left(x_{1}, y_{1}\right)}{\Psi\left(x_{1}, y_{1}\right)}=\frac{\Phi\left(x^{*}, y_{0}\right)}{\Psi\left(x^{*}, y_{0}\right)} .
$$

If $x_{1} \neq x^{*}$, we could deduce

$$
\phi\left(x_{1}\right)>\frac{\Phi\left(x^{*}, y_{0}\right)}{\Psi\left(x^{*}, y_{0}\right)}
$$


which would contradict (5.11).

Suppose (5.12) does not hold, then $\phi\left(x_{1}\right) \leq \Phi\left(x^{*}, y_{0}\right) / \Psi\left(x^{*}, y_{0}\right)$, that is,

$$
\frac{\Phi\left(x^{*}, y_{0}\right)}{\Psi\left(x^{*}, y_{0}\right)} \geq \phi\left(x_{1}\right)=\max _{y \in I} \frac{\Phi\left(x_{1}, y\right)}{\Psi\left(x_{1}, y\right)} \geq \frac{\Phi\left(x_{1}, y_{0}\right)}{\Psi\left(x_{1}, y_{0}\right)}
$$

or $D\left(x_{1}\right)=\Phi\left(x_{1}, y_{0}\right) \Psi\left(x^{*}, y_{0}\right)-\Phi\left(x^{*}, y_{0}\right) \Psi\left(x_{1}, y_{0}\right) \leq 0=D\left(x^{*}\right)$, that is,

$$
D\left(x_{1}\right) \leq D\left(x^{*}\right) .
$$

Since $D$ is strictly pseudoinvex, (5.13) implies $D^{\prime}\left(x^{*}\right) \eta\left(x_{1}, x^{*}\right)<0$, or

$$
\left[\Psi\left(x^{*}, y_{0}\right) \Phi_{1}^{\prime}\left(x^{*}, y_{0}\right)-\Phi\left(x^{*}, y_{0}\right) \Psi_{1}^{\prime}\left(x^{*}, y_{0}\right)\right] \eta\left(x_{1}, x^{*}\right)<0 .
$$

By (5.1) and (5.14), we get $-\Psi\left(x^{*}, y_{0}\right) \Omega_{1}^{\prime}\left(x^{*}, z_{0}\right) \eta\left(x_{1}, x^{*}\right)<0$, that is,

$$
\Omega_{1}^{\prime}\left(x^{*}, z_{0}\right) \eta\left(x_{1}, x^{*}\right)>0,
$$

since $\Psi\left(x^{*}, y_{0}\right)>0$. By the quasi-invexity of $\Omega,(5.15)$ implies

$$
\Omega\left(x_{1}, z_{0}\right)>\Omega\left(x^{*}, z_{0}\right) .
$$

On the other hand, $x_{1} \in \mathscr{F}_{P}, z_{0} \in R_{+}^{m}$ and $\Omega\left(x_{1}, z_{0}\right) \leq 0$. By (5.2), $\Omega\left(x^{*}, z_{0}\right) \geq 0$. Thus $\Omega\left(x_{1}, z_{0}\right) \leq \Omega\left(x^{*}, z_{0}\right)$ which contradicts (5.16). Hence (5.12) holds under $x_{1} \neq x^{*}$. Therefore $x_{1}=x^{*}$.

\section{The third dual model}

In this section, we employ Theorems 2.2 and 3.1 to construct a one-parameter dual problem for $(P)$, and consider

$$
\begin{aligned}
& \text { (D3) Maximise } v \\
& \text { subject to }(u, v, y, z) \in P S\left(T, R^{n}\right) \times R_{+} \times I \times R_{+}^{m} \text {, } \\
& u(a)=\alpha, \quad u(b)=\beta, \\
& \Phi_{1}^{\prime}(u, y)-v \Psi_{1}^{\prime}(u, y)+\Omega_{1}^{\prime}(u, z)=0, \\
& \Phi(u, y)-v \Psi(u, y) \geq 0, \quad \Omega(u, z) \geq 0 .
\end{aligned}
$$

Denote by $K_{3}$ the set of all feasible solutions of problem (D3). Then by a proof similar to that for Theorems 5.1-5.3, we can obtain the following theorems relating $(P)$ and (D3). 
THEOREM 6.1 (Weak duality). Let $x \in \mathscr{F}_{P},(u, v, y, z) \in K_{3}$. Define

$$
N(x)=\Phi(x, y)-v \Psi(x, y) \text { and } M(x)=N(x)+\Omega(x, z) .
$$

If, for $y \in I, z \in R_{+}^{m}$, any one of the following conditions holds:

(a) $\Phi(\cdot, y),-\Psi(\cdot, y)$ and $\Omega(\cdot, z)$ are invex w.r.t. the function $\eta$;

(b) $N$ is pseudoinvex and $\Omega(\cdot, z)$ is quasi-invex w.r.t. $\eta$;

(c) $N$ is quasi-invex and $\Omega(\cdot, z)$ is strictly pseudoinvex w.r.t. $\eta$;

(d) $M$ is pseudoinvex w.r.t. $\eta$,

then $\phi(x) \geq v$.

THEOREM 6.2 (Strong duality). If $x^{*}$ is an optimal solution of $(P)$ satisfying the conditions of Theorems 2.1 and 6.1 , then there exist $y^{*} \in I, z 1^{*} \in R_{+}^{m}$ and $v^{*} \in R_{+}$, such that $\left(x^{*}, y^{*}, z^{*}, v^{*}\right)$ is an optimal solution of $(D 3)$, and $(P)$ and $(D 3)$ have the same optimal values.

THEOREM 6.3 (Strict converse duality). Let $x_{1}$ and $\left(x^{*}, y_{0}, z_{0}, v_{0}\right)$ be optimal solutions of $(P)$ and $(D 3)$, respectively, and let the conditions of Theorem 6.2 be fulfilled. If $L(\cdot)=\Phi\left(\cdot, y_{0}\right)-v_{0} \Psi\left(\cdot, y_{0}\right)$ is strictly pseudoinvex and $\Omega\left(\cdot, z_{0}\right)$ is quasi-invex w.r.t. the function $\eta$, then $x_{1}=x^{*}$ is an optimal solution of $(P)$, and $(P)$ and $(D 3)$ have the same optimal values $\phi\left(x_{1}\right)=v_{0}$.

It is remarkable that most of the fractional objectives other than the integral expression can also be employed technically as demonstrated in this paper.

\section{Acknowledgement}

The authors wish to express their deep gratitude to Professor B. D. Craven for his valuable comments on an earlier version of the paper, and in particular for his observation that the definition of invexity on integral expressions coincides with the original concept of invexity, thus simplifying many processes. This research has been partly supported by NSC, Taiwan.

\section{References}

[1] C. R. Bector, S. Chandra and I. Husain, "Generalized continuous fractional programming duality: a parametric approach", Util. Math. 42 (1992) 39-60.

[2] C. R. Bector, S. Chandra and V. Kumar, "Duality for minimax programming involving $V$-invex functions", Optimization 30 (1994) 93-103. 
[3] C. R. Bector and S. K. Suneja, "Duality in nondifferentiable generalized fractional programming", Asia-Pacific J. Oper. Res. 5 (1988) 134-139.

[4] S. Chandra, B. D. Craven and I. Husain, "A class of nondifferentiable continuous programming problems", J. Math. Anal. Appl. 107 (1985) 122-131.

[5] S. Chandra, B. D. Craven and I. Husain, "Continuous programming containing arbitrary norms", J. Austral. Math. Soc. Ser. A 39 (1985) 28-38.

[6] S. Chandra, B. D. Craven and B. Mond, "Generalized fractional programming duality: a ratio game approach", J. Austral. Math. Soc. Ser. B 28 (1986) 170-180.

[7] B. D. Craven, "Lagrangian conditions for a minimax", in Workshop/Miniconference on Functional Analysis and Optimization (July 8-24, 1988), (Proc. Center Math. Anal., Australian National University, 1988) 24-33.

[8] B. D. Craven, "On continuous programming with generalized convexity", Asia-Pacific J. Oper. Res. 10 (1993) 219-231.

[9] J.-P. Crouzeix, J. A. Ferland and S. Schaible, "Duality in generalized fractional programming", Math. Prog. 27 (1983) 342-354.

[10] M. A. Hanson and B. Mond, "Further generalizations of convexity in mathematical programming", J. Inf. Optim. Sci. 3 (1982) 25-32.

[11] H. C. Lai and J. C. Liu, "Optimality conditions for multiobjective programming with generalized ( $\Im, \rho, \theta)$-convex set functions", J. Math. Anal. Appl. 215 (1997) 443-460.

[12] H. C. Lai and J. C. Liu, "Duality for a minimax programming problem containing $n$-set functions", J. Math. Anal. Appl. 229 (1999) 587-604.

[13] H. C. Lai and J. C. Liu, "On minimax fractional programming of generalized convex set functions", J. Math. Anal. Appl. 244 (2000) 442-405.

[14] H. C. Lai, J. C. Liu and K. Tanaka, "Duality without a constraint qualification for minimax fractional programming", J. Optim. Theory Appl. 101 (1999) 91-102.

[15] H. C. Lai, J. C. Liu and K. Tanaka, "Necessary and sufficient conditions for minimax fractional programming", J. Math. Anal. Appl. 230 (1999) 311-328.

[16] J. C. Liu, "Optimality and duality for multiobjective fractional programming involving nonsmooth $(f, \rho)$-convex functions", Optimization 36 (1996) 333-346.

[17] J. C. Liu, "Optimality and duality for multiobjective fractional programming involving nonsmooth $(f, \rho)$-convex functions", Optimization 37 (1996) 369-383.

[18] B. Mond, S. Chandra and I. Husain, "Duality for variational problems with invexity", J. Math. Anal. Appl. 134 (1988) 322-328.

[19] B. Mond and 1. Husain, "Sufficient optimality criteria and duality for variational problems with generalized invexity", J. Austral. Math. Soc. Ser. B 31 (1989) 108-121.

[20] I. M. Stancu-Minasian, Fractional programming theory, methods and applications (Kluwer, Dordrecht, 1997).

[21] G. J. Zalmai, "Optimality conditions and duality for a class of continuous-time generalized fractional programming problems", J. Math. Anal. Appl. 153 (1990) 356-371.

[22] G. J. Zalmai, "Optimality conditions and duality models for generalized fractional programming problems containing locally subdifferentiable and $\rho$-convex functions", Optimization 32 (1995) 95-124. 